\title{
Systemic immune-inflammation index predicting chemoradiation resistance and poor outcome in patients with stage III non-small cell lung cancer
}

\author{
Yu-Suo Tong ${ }^{1}$, Juan Tan ${ }^{2}$, Xi-Lei Zhou ${ }^{1}$, Ya-Qi Song ${ }^{1}$ and Ying-Jian Song ${ }^{3 *}$
}

\begin{abstract}
Background: There is increasing evidence that the existence of systemic inflammation response is correlated with poor prognosis in several solid tumors. The aim of this retrospective study was to investigate the association between systemic immune-inflammation index (SII) and therapy response and overall survival in patients with stage III nonsmall cell lung cancer (NSCLC). The prognostic values of neutrophil to lymphocyte ratio (NLR), platelet to lymphocyte ratio (PLR), and prognostic nutritional index (PNI) were also evaluated.
\end{abstract}

Methods: In total, 332 patients with new diagnosis of stage III NSCLC were included in this retrospective analysis. SII was defined as platelet counts $\times$ neutrophil counts/lymphocyte counts. Receiver operating characteristic (ROC) curve was used to evaluate the optimal cut-off value for SII, NLR, PLR and PNI. Univariate and multivariate survival analysis were performed to identify the factors correlated with overall survival.

Results: Applying cut-offs of $\geq 660$ (SII), $\geq 3.57$ (NLR), $\geq 147$ (PLR), $\leq 52.95$ (PNI), SII $\geq 660$ was significantly correlated with worse ECOG PS $(<0.001)$, higher T stage $(<0.001)$, advanced clinical stage $(p=0.019)$, and lower response rate ( $p=0.018$ ). In univariate analysis, SII $\geq 660, N L R \geq 3.57, P L R \geq 147$, and PNI $\leq 52.95$ were significantly associated with worse overall survival ( $p_{\text {all }}<0.001$ ). Patients with $\mathrm{SII} \geq 660$ had a median overall survival of 10 months, and patients with SII < 660 showed a median overall survival of 30 months. In multivariate analysis only ECOG PS (HR, 1.744; $95 \%$ Cl 1.158-2.626; $p=0.008)$, T stage ( $H R, 1.332 ; 95 \% \mathrm{Cl} 1.032-1.718 ; p=0.028)$, N stage (HR, 1.848; 95\% Cl 1.113-3.068; $p=0.018)$, SII (HR, 2.105; 95\% Cl 1.481-2.741; $p<0.001)$ and NLR $\geq 3.57(\mathrm{HR}, 1.934 ; 95 \% \mathrm{Cl} 1.448-2.585 ; p<0.001)$ were independently correlated with overall survival.

Conclusions: This study demonstrates that the SII is an independent prognostic indicator of poor outcomes for patients with stage III NSCLC and is superior to other inflammation-based factors in terms of prognostic ability.

Keywords: Non-small cell lung cancer, prognostic factor, Systemic immune-inflammation index, Chemoradiation

\section{Background}

Lung cancer remains the leading cause of cancer-related mortality in the world, accounting for 1.3 million deaths each year [1]. Non-small cell lung cancer (NSCLC) compromises more than $85 \%$ of all lung cancers cases [2].

\footnotetext{
*Correspondence: songyingjianhuaian@163.com

${ }^{3}$ Department of Respiratory Medicine, Huai'an First People's Hospital,

Nanjing Medical University, Huai'an, Jiangsu, China

Full list of author information is available at the end of the article
}

Approximately $20-25 \%$ patients with NSCLC are diagnosed with locally advanced disease (stage III) and have poor survival [3]. For these patients, two standard treatment options are offered: the concurrent chemoradiotherapy (CRT) or induction chemotherapy followed by surgery $[4,5]$. However, even after complete resection and postoperative consolidation chemotherapy, $20-40 \%$ patients still have a risk of local recurrence [6]. Indeed, NSCLC is poorly chemosensitive to most of the available agents, the reported treatment response rates is only $10-25 \%$ [7]. 
Chemotherapy resistance and development of local recurrence or distant metastases are the main obstacles in the treatment of locally advanced NSCLC. Therefore, identification of prognostic factors that can be used to predict treatment response or long-term survival is required.

It is widely recognized that systemic inflammation plays an important role in the development and progression of many solid tumors [8]. The existence of systemic inflammation, as measured by parameters such as neutrophil to lymphocyte ratio (NLR), platelet to lymphocyte ratio (PLR), and prognostic nutritional index (PNI) was reported to be correlated with poor prognosis across multiple malignancies, including NSCLC [913]. Recently, the systemic immune-inflammation index (platelet counts $\times$ neutrophil counts/lymphocyte counts, SII) has been shown to have independent prognostic value in patients with hepatocellular carcinoma treated with surgery [14]. However, the clinical implication of SII in chemoradiotherapy resistance and survival in locally advanced NSCLC remains largely unknown.

The present study had three aims: first, to evaluate the prognostic significance of SII in patients with locally advanced NSCLC treated with primary chemoradiotherapy, second, to investigate whether SII was able to predict treatment response to chemoradiotherapy. Finally, to compare the prognostic values of inflammation-based prognostic factors (NLR, PLR, and PNI) with SII.

\section{Methods}

\section{Patient section}

The study was approved by the medical ethics committee of our institute, and informed consent was exempted due to the retrospective nature of the study.

Consecutive patients who were newly diagnosed with stage III NSCLC between January 2006 and May 2012 in our hospital (Affiliated Huai'an First Hospital, Nanjing Medical University, Jiangsu, China) were collected in the present study. All medical records were reviewed retrospectively. Patients who met the following inclusion criteria were selected: (a) biopsy proven NSCLC; (b) stage III A or stage III B disease according to the 6th edition of tumor-node-metastasis (TNM) classification; (c) Eastern Cooperative Oncology Group performance status (ECOG PS) (0-2); (d) $\leq 70$ years of age; (e) treatment with concurrent CRT or surgery followed by chemoradiotherapy. Patients with hematologic malignancies, chronic inflammatory disease, or clinical evidence of acute infection were excluded. Patients who received neoadjuvant chemotherapy were also excluded.

\section{Data collection and definition}

Patient characteristics including age, sex, history of tobacco exposure, pathologic type, TNM stage, full blood count, ECOG PS and the details of treatment were collected by electronic medical reports. Full blood counts were obtained before the initiation of any treatment (surgery, radiation, or chemotherapy). The SII, NLR, PLR and PNI were calculated as follows: SII $=$ platelet counts $\times$ neutrophil counts/lymphocyte counts, NLR $=$ neutrophil count/lymphocyte count, $\mathrm{PLR}=$ platelet count/lymphocyte count, $\mathrm{PNI}=$ albumin $(\mathrm{g} / \mathrm{L})+5 \times$ total lymphocyte count $\left(10^{9} / \mathrm{L}\right)$.

\section{Treatment details}

\section{Surgery-chemoradiotherapy group}

All patients underwent tumor resection and systemic lymph node dissection. 4-6 weeks after surgery, cisplatin-based adjuvant chemotherapy was performed every 3 weeks. 2-4 cycles of chemotherapy were administered according the decisions of the physicians. If there was incomplete resection (RI or R2) after surgery, patients received postoperative radiotherapy (1.8-2.0 Gy/day, 5 days/week, 50.4-66 Gy).

\section{CRT group}

Concurrent radiotherapy using 6 or $15 \mathrm{MV}$ X-rays was delivered at a dose of 1.8-2.0 Gy/day, 5 days/week, with a total radiation dose of 40-66 Gy. Chemotherapy started on day 1 , concurrent with the beginning of radiation. Most patients received four to six cycles of cisplatinbased concurrent chemotherapy.

\section{Response assessment and follow-up}

One month after complete of treatment, tumor response was evaluated by $\mathrm{CT}$ scan according to the Response Evaluate Criteria for Solids Tumors (RECIST). Complete response (CR) was defined as total regression of all assessable lesions; partial response (PR) was defined as the disappearance of at least of $30 \%$ in the sum of the longest diameters of the target lesions; progressive disease (PD) was defined as more than a $20 \%$ increase in primary tumor volume or appearance of new lesions; the remaining patients which did not meet the criteria of $\mathrm{PD}$ or PR were categorized as stable disease (SD) [15]. The objective response rates were calculated by the percentage of CR and PR among all treated patients.

Patients were followed every 3 months for the first year, then every 6 months for 2 years, and then every year or until death. Follow-up data were obtained from patient medical records and telephone interview.

\section{Statistical analysis}

All statistical analyses were performed using SPSS software (version 20.0). Receiver operating characteristic (ROC) curves were used to calculate the optimal cut-off value for SII, NLR, PLR and PNI, and the end-point was 
based on overall survival (OS) in the study. Categorical variables were reported as frequencies and percentages, and were analyzed using Fisher's exact tests or Chi square tests. OS was defined as the time from diagnosis to date of death due to any cause. Data from patients who were alive by the time of analysis were censored. Survival analyses were performed using Kaplan-Meier method. The differences between the survival curves were compared by using Log rank test. The multivariate Cox hazard regression analysis was performed on the factors that were shown to be significant on univariate analysis. All tests were two-sided and $p$ values less than 0.05 were considered significant.

\section{Results}

\section{Patient characteristics}

Between January 2006 and May 2012, a total of 545 patients with stage III NSCLC were initially identified, of whom 332 patients were eligible for analysis. Of the included patients, 115 (35\%) patients underwent surgical resection followed by chemotherapy or chemoradiotherapy, the remaining patients $(\mathrm{n}=217,65 \%)$ received concurrent CRT. Baseline patient characteristics are summarized in Table 1. The median age was 61 (range 34-70) years, $206(62 \%)$ patients were male and $126(38 \%)$ were female. Among these patients, 197 (59.3\%) had stage IIIA UICC-6 disease, and 135 (40.7\%) had stage IIIB UICC-6 disease. Approximate half of the patients $(n=154,46.4 \%)$ were determined to have adenocarcinoma, 161 (48.5\%) had squamous cell carcinoma, 17 (5.1\%) had adenosquamous carcinoma, or other histology.

In the surgery-chemoradiotherapy group, all patients underwent surgical resection followed by cisplatin-based doublet chemotherapy. The median number of chemotherapy cycle was four (range 2-4 cycles). For patients with R1 or R2 resection, postoperative radiation therapy was administered in 47 patients. In the concurrent CRT group, all patients received cisplatin-based concurrent chemotherapy, including cisplatin in combination with etoposide $(n=23)$, cisplatin plus docetaxel $(n=96)$, cisplatin plus paclitaxel $(\mathrm{n}=54)$ and cisplatin plus vinorebine $(\mathrm{n}=44)$. After concurrent CRT, 171 patients $(78.8 \%)$ revived four courses of consolidation chemotherapy. The most commonly used chemotherapy regimen for consolidation was docetaxel and cisplatin.

At baseline, the median values of SII, NLR, PLR and PNI for all study population were 634.14 (range 159.804299.90), 3.05 (range 0.59-19.28), 141.29 (range 34.63571.79 ) and 50.31 (range 15.59-67.75), respectively.

\section{Tumor response to CRT and patient outcomes}

Of the 217 patients who received concurrent CRT, CR, PR, SD and PD were observed in 12 (5.5\%), 137 (63.2\%),

\section{Table 1 Baseline patient characteristics}

\begin{tabular}{|c|c|}
\hline Characteristics & Number (\%) \\
\hline \multicolumn{2}{|l|}{ Age } \\
\hline Median & 61 \\
\hline Range & $34-70$ \\
\hline \multicolumn{2}{|l|}{ Gender } \\
\hline Male & $206(62 \%)$ \\
\hline Female & $126(38 \%)$ \\
\hline \multicolumn{2}{|l|}{ Smoking status } \\
\hline Never smoker & $141(42.5 \%)$ \\
\hline Current or ex-smoker & $191(57.5 \%)$ \\
\hline \multicolumn{2}{|l|}{ ECOG PS } \\
\hline $0-1$ & $304(91.6 \%)$ \\
\hline 2 & $28(8.4 \%)$ \\
\hline \multicolumn{2}{|l|}{ Histological subtype } \\
\hline Adenocarcinoma & $154(46.4 \%)$ \\
\hline Squamous & $161(48.5 \%)$ \\
\hline Other histology & $17(5.1 \%)$ \\
\hline \multicolumn{2}{|l|}{ T stage } \\
\hline $\mathrm{T} 1$ & $59(17.8 \%)$ \\
\hline $\mathrm{T} 2$ & $122(36.7 \%)$ \\
\hline T3 & $74(22.3 \%)$ \\
\hline T4 & $77(23.2 \%)$ \\
\hline \multicolumn{2}{|l|}{ N stage } \\
\hline NO & $24(7.2 \%)$ \\
\hline N1 & $29(8.7 \%)$ \\
\hline $\mathrm{N} 2$ & $221(66.6 \%)$ \\
\hline N3 & $58(17.5 \%)$ \\
\hline \multicolumn{2}{|l|}{ Clinical stage } \\
\hline III A & $197(59.3 \%)$ \\
\hline III B & $135(40.7 \%)$ \\
\hline \multicolumn{2}{|l|}{ Treatment modality } \\
\hline Surgery + chemoradiation & $115(34.6 \%)$ \\
\hline Concurrent chemoradiation & $217(65.4 \%)$ \\
\hline \multicolumn{2}{|l|}{ Chemotherapy cycles } \\
\hline Median & 4 \\
\hline Range & $2-8$ \\
\hline \multicolumn{2}{|l|}{ Chemotherapy regimen utilized } \\
\hline Cisplatin + etoposide & $36(10.8 \%)$ \\
\hline Cisplatin + docetaxel & $150(45.2 \%)$ \\
\hline Cisplatin + paclitaxel & $61(18.4 \%)$ \\
\hline Cisplatin + vinorebine & $47(14.2 \%)$ \\
\hline Cisplatin + others & $38(11.4 \%)$ \\
\hline
\end{tabular}

$64(29.5 \%)$ and $4(1.8 \%)$ cases, respectively. The objective response rates were $68.7 \%(149 / 217)$.

With a median follow-up time of 22 months (range 2-72 months), 281 (84.6\%) patients had died and 51 (15.4\%) patients were living at the end of the follow-up period. The 1-, 3- and 5-year OS of all study population were $65.4,28.6,15.4 \%$, respectively. 


\section{Selection of optimal cut-off values for SII, NLR, PLR, and PNI}

Different studies have suggested different cut-off values when analyzing SII, NLR, PLR and PNI in prognostic setting. We therefore attempted to establish the optimal thresholds for these biomarkers on our study population through ROC curve analysis. As shown in Fig. 1, the area under the curves (AUC) for OS were $0.673(p<0.001)$, $0.604(p=0.019), 0.603(p=0.018)$ and $0.621(p=0.006)$ for SII, NLR, PLR and PNI, respectively. The optimal cut-off values for the prediction of OS by ROC analysis was 660 for SII, 3.57 for NLR, 147 for PLR and 52.95 for PNI. Consequently, patients were separately divided into two groups with high or low levels according to the optimal cut-off values. One hundred and forty-nine patients $(44.9 \%)$ had SII $\geq 660,137$ patients $(41.3 \%)$ had NLR $\geq 3.57,153$ patients (46.1\%) had PLR $\geq 147$ and 94 (28.3\%) patients had PNI $\geq 52.95$.

\section{Association of inflammation-based factors with clinicopathological characteristics}

The relationship between SII, NLR, PLR and PNI and patient characteristics is shown in Table 2. Using the criteria described earlier, patients with SII $\geq 660$ were more likely to have worse ECOG PS $(p<0.001)$, higher tumor $(\mathrm{T})$ stage $(p<0.001)$ and advanced clinical stage $(p=0.019)$ than those with SII $<660$. In addition, patients with high NLR and PLR had statistically significantly advanced clinical stage compared those with low NLR and PLR ( $p=0.035$ and $<0.001$, respectively).

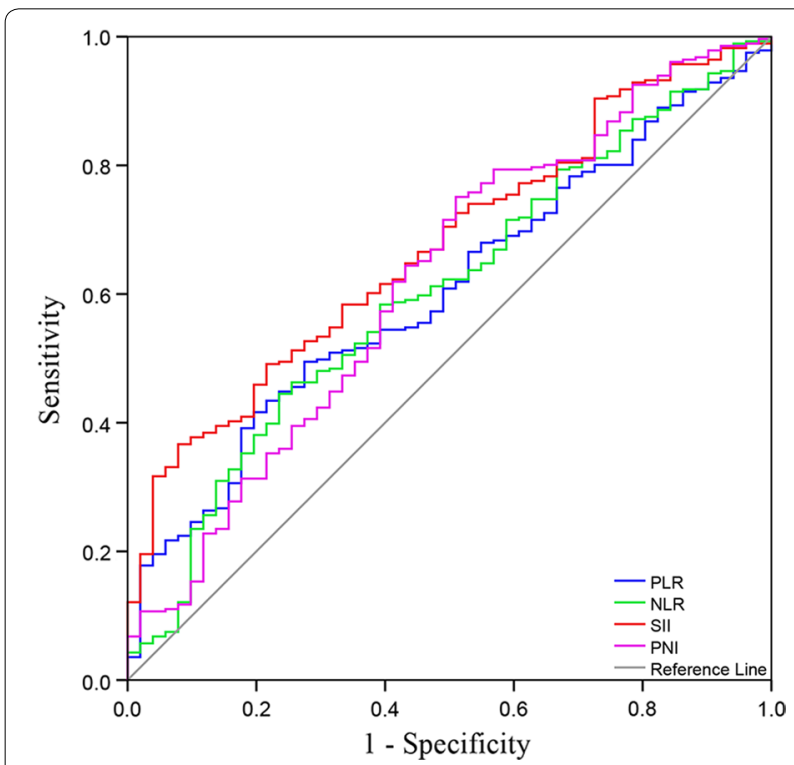

Fig. 1 Receiver operating characteristic curve analysis for the optimal cut-off value of SII, NLR, PLR and PNI. The areas under the curve for overall survival were $0.673,0.603,0.604$ and 0.621 for SII, NLR, PLR and $\mathrm{PNI}$, respectively
Patients with tobacco use also had higher NLR $(p=0.006)$. By contrast, patients with high PNI were significantly younger $(p=0.037)$ and have better ECOG PS $(p=0.031)$ than those with low PNI. Moreover, PNI was significantly higher in patients diagnosed with stage III A cancer compared with those with stage III B disease $(p=0.022)$.

\section{Baseline SII and response to treatment}

A total of 217 patients underwent concurrent CRT. In patients who were SII $\geq 660$ ( $\mathrm{n}=118,54.4 \%)$, CR, PR, $\mathrm{SD}$ and PD were observed in 3 (2.6\%), 70 (59.3\%), 43 (36.4\%) and $2(1.7 \%)$ cases, respectively. However, in patients with SII < $660(\mathrm{n}=99,45.6 \%), \mathrm{CR}, \mathrm{PR}, \mathrm{SD}$ and PD were achieved in 9 (9.1\%), 67 (67.7\%), 21 (21.2\%) and $2(2 \%)$ patients, respectively. Thereafter, patients with SII < 660 had significantly higher response rate to treatment than those with SII $\geq 660$ (76.8\% vs $61.9 \%$, $p=0.018$ ). However, NLR, PLR, and PNI did not show any significant correlation with treatment response $\left(p_{\text {all }}>0.05\right)$.

\section{Prognostic value of SII, NLR, PLR and PNI and other clinicopathological factors}

The correlation between inflammation-based factors and OS is shown in Fig. 2. Patients with SII $\geq 660$, NLR $\geq 3.57$, PLR $\geq 147$ and PNI $<52.95$ had significantly worse OS $\left(p_{\text {all }}<0.05\right)$. Patients with SII $\geq 660$ had a median OS of 10 months whereas patients with SII $<660$ showed a median OS of 30 months. In addition, patients with NLR $\geq 3.57$ had a median OS of 10 months, compared with 28 months for patients with NLR $<3.57$. The median OS was 11 months for patients with PLR $\geq 147$ and 27 months for patients with PLR $<147$. And the median OS was 15 months for patients with PNI $<52.95$ and 27 months for patients with PNI $\geq 52.95$. SII provided the greatest survival difference with a 5-year OS rate of $21.9 \%$ in SII $<660$ vs $7.4 \%$ in SII $\geq 660$, followed by PNI $(25.5 \%$ PNI $\geq 52.95$ vs $11.3 \%$ PNI $<52.95)$, and then PLR $(20.7 \%$ PLR $<147$ vs $9.2 \%$ PLR $\geq 147)$, and NLR $(20 \%$ NLR $<3.57$ vs $8.8 \%$ NLR $\geq 3.57)$.

However, in this analysis, SII $\geq 660$ group contained many patients $(47.7 \%)$ who underwent concurrent CRT for stage III B disease compared with patients in SII $<660$ group (30.3\%), which may influence the results. The prognostic value of SII was next investigated in stage III A and stage III B subgroup, separately. As shown in Fig. 3, patients with SII $\geq 660$ still have worse OS in both stage III A and stage III B disease. Separate analyses the prognostic value of SII, NLR, PLR and PNI in patients with adenocarcinoma and squamous cell carcinoma also showed significant effects in both groups $\left(p_{\text {all }}<0.05\right.$, data not shown). 


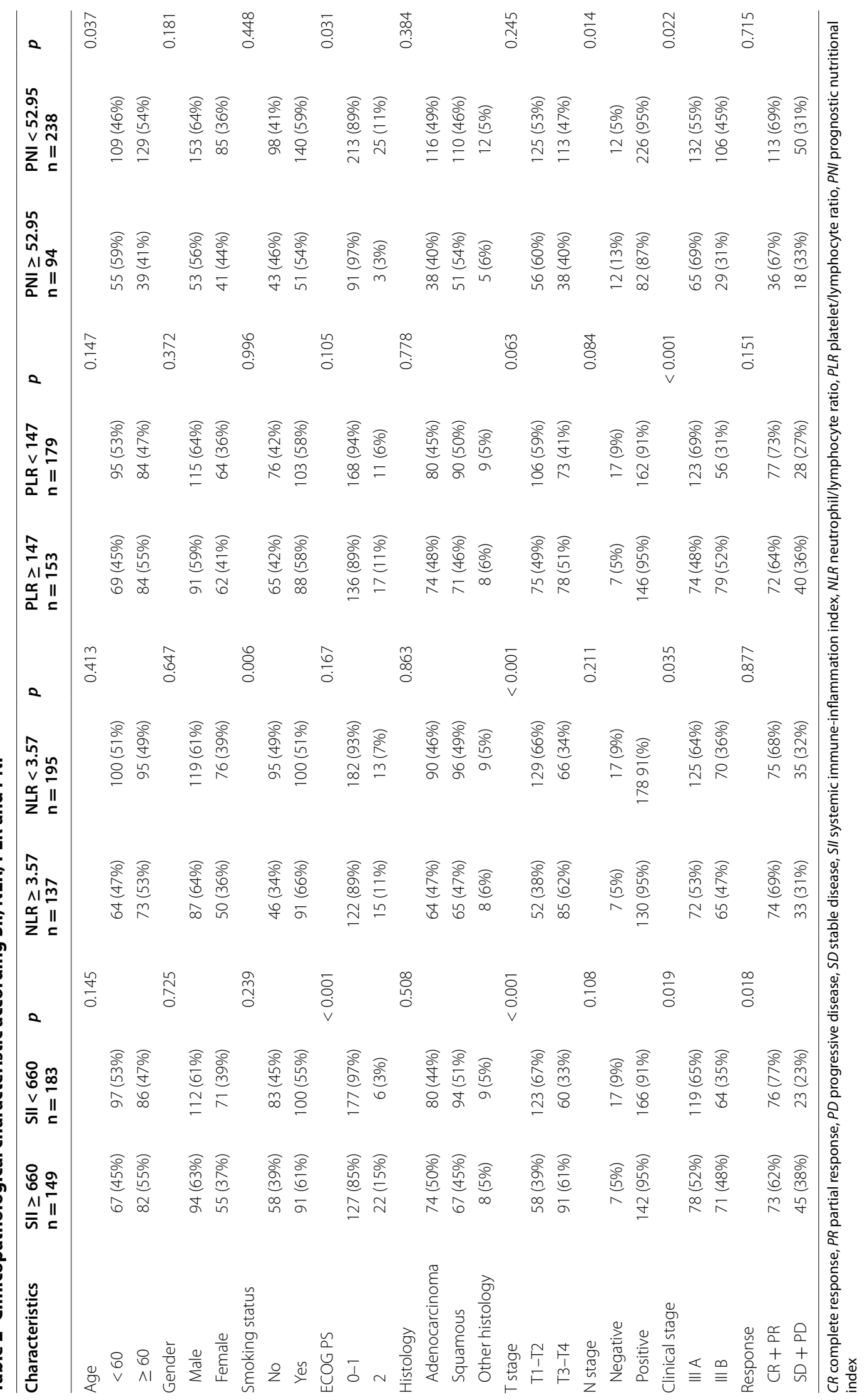



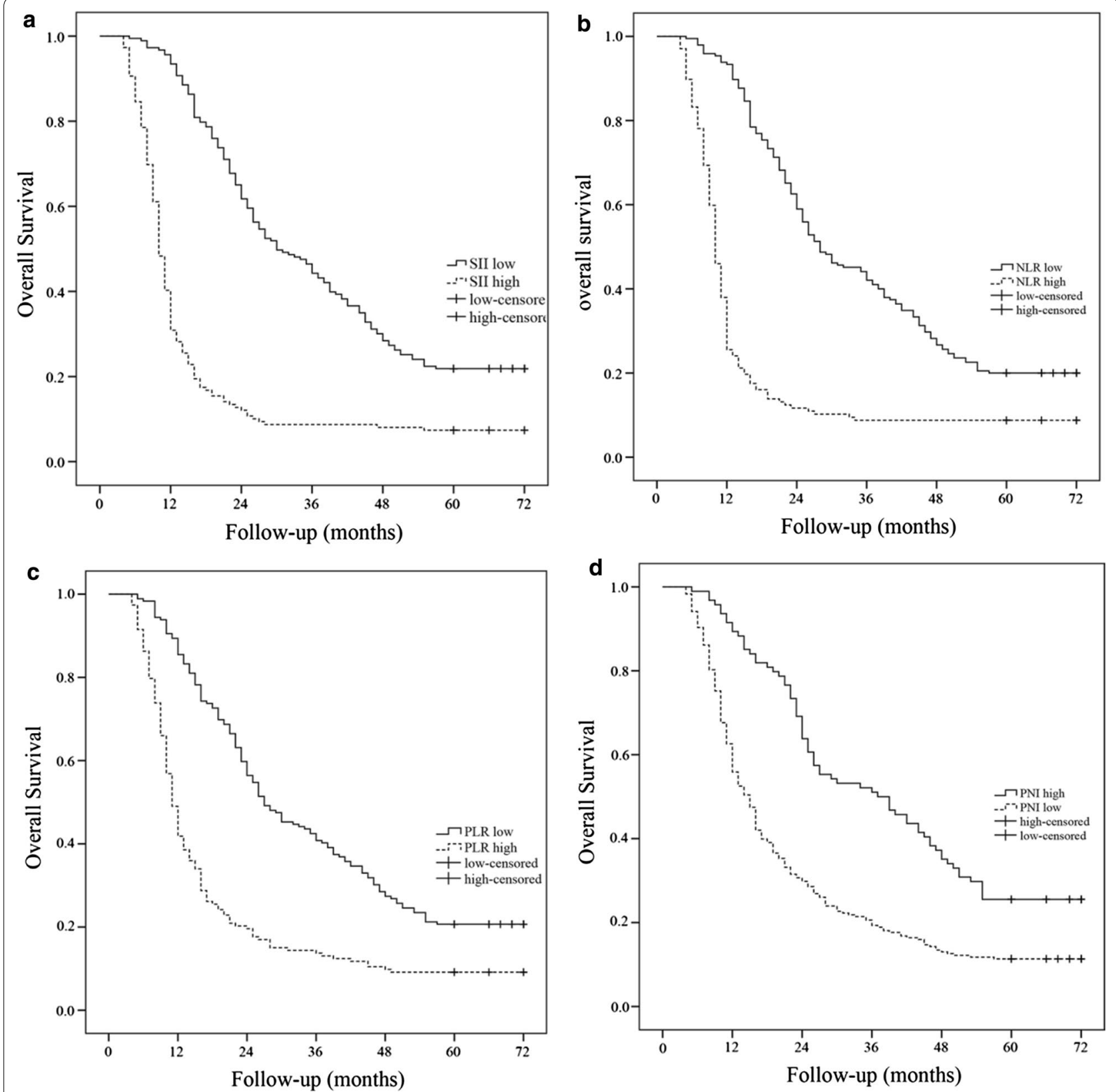

Fig. 2 Kaplan-Meier curves of overall survival according to SII (a), NLR (b), PLR (c) and PNI (d)

On univariate cox regression analyses, ECOG PS $(p<0.001), \mathrm{T}$ stage $(p=0.002)$, node $(\mathrm{N})$ stage $(p=0.028)$, clinical stage $(p=0.034)$, SII $(p<0.001)$, $\operatorname{NLR}(p<0.001)$, PLR $(p<0.001)$ and PNI $(p<0.001)$ were significantly correlated with OS (Table 3 ). All 8 clinicopathological characteristics were further investigated in multivariate analysis. As shown in Table 4, SII (HR, 2.105; 95\% CI 1.481-2.741; $p<0.001$ ) was the most significantly independent predictor of worse OS, followed by NLR (HR, 1.934; 95\% CI 1.448-2.585; $p<0.001$ ). Meanwhile,
ECOG PS (HR, 1.744; 95\% CI 1.158-2.626; $p=0.008$ ), $\mathrm{T}$ stage (HR, 1.332; 95\% CI 1.032-1.718; $p=0.028)$ and $\mathrm{N}$ stage (HR, 1.848; 95\% CI 1.113-3.068; $p=0.018$ ) were also independent risk factors for OS.

\section{Discussion}

In the present study, we evaluated prognostic value of inflammation-based factors (SII, NLR, PLR and PNI) in patients with stage III NSCLC treated with primary chemoradiotherapy to identify patients who could 

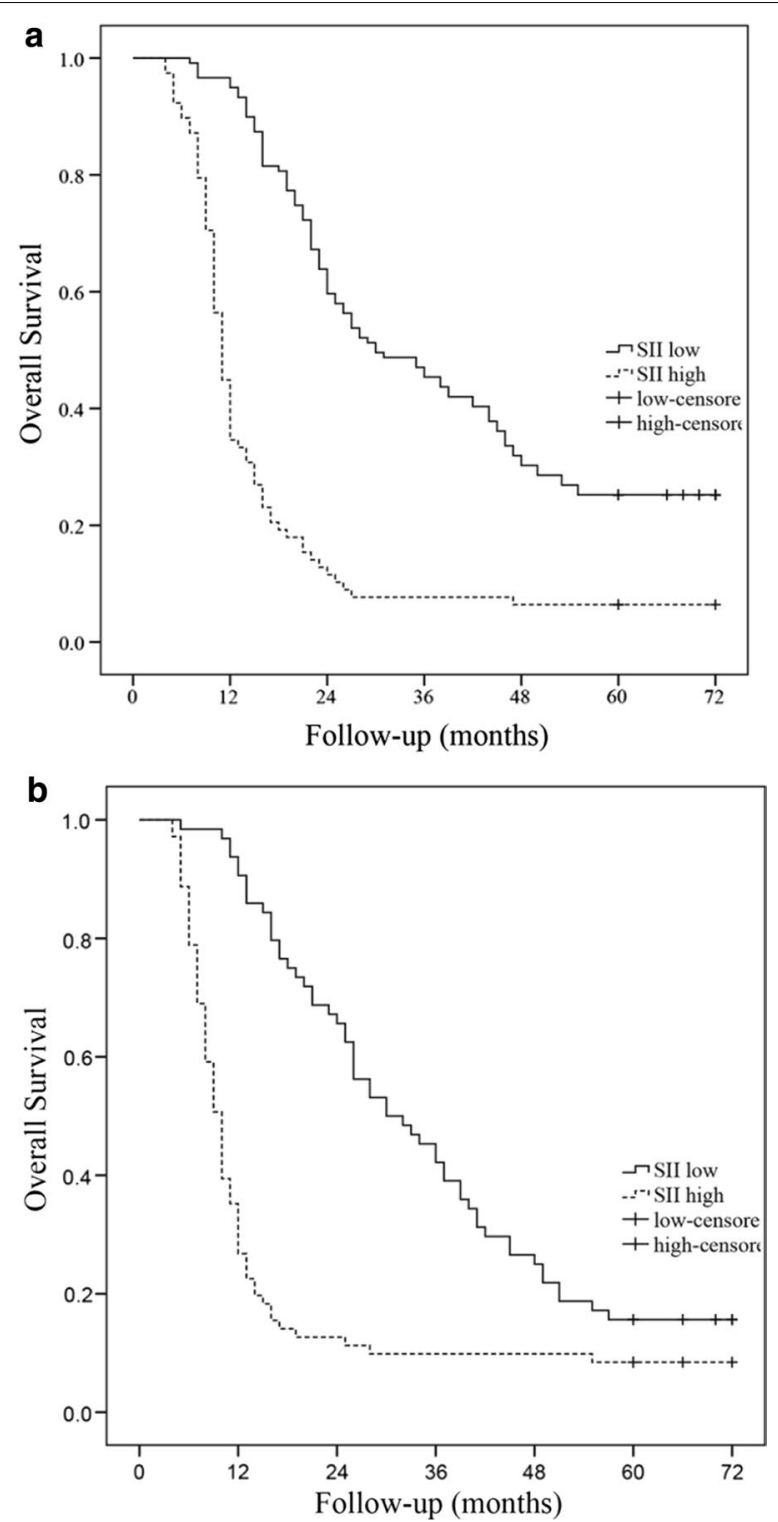

Fig. 3 Kaplan-Meier survival analysis in stage III A (a) and stage III B (b) NSCLC subgroup

benefit from current treatment. We found that patients with SII $\geq 660$ were more likely to have higher $\mathrm{T}$ stage, worse ECOG PS, advanced clinical stage and lower response rate than patients with SII $<660$. Furthermore, the pre-treatment SII was also found to be an independent prognostic biomarker for OS and was superior to NLR, PLR and PNI in terms of prognostic ability.

Recently, several studies have revealed that inflammation-based factors are correlated with aggressive tumor characteristics in various tumors. In the study by Deng et al., NLR and PLR were significantly associated with tumor stage, deep of invasion, and lymph node metastasis
Table 3 Univariate analysis of potential factors associated with overall survival in patients with locally advanced NSCLC

\begin{tabular}{|c|c|c|c|}
\hline Variables & Case & $\begin{array}{l}\text { Overall survival } \\
\text { Median (months) }\end{array}$ & $p^{*}$ \\
\hline Age & & & 0.691 \\
\hline$<60$ & 164 & 21 & \\
\hline$\geq 60$ & 168 & 17 & \\
\hline Gender & & & 0.492 \\
\hline Male & 206 & 18 & \\
\hline Female & 126 & 19 & \\
\hline Smoking status & & & 0.154 \\
\hline Never smoker & 141 & 21 & \\
\hline Current or ex-smoker & 191 & 16 & \\
\hline ECOG PS & & & $<0.001$ \\
\hline $0-1$ & 304 & 21 & \\
\hline 2 & 28 & 12 & \\
\hline Histological subtype & & & 0.654 \\
\hline Adenocarcinoma & 154 & 18 & \\
\hline Squamous & 161 & 21 & \\
\hline Other histology & 17 & 14 & \\
\hline T stage & & & 0.002 \\
\hline $\mathrm{T} 1-\mathrm{T} 2$ & 181 & 22 & \\
\hline T3-T4 & 151 & 14 & \\
\hline N stage & & & 0.028 \\
\hline Negative & 24 & 42 & \\
\hline Positive & 308 & 17 & \\
\hline Clinical stage & & & 0.034 \\
\hline III A & 197 & 21 & \\
\hline$\| I I B$ & 135 & 15 & \\
\hline Chemotherapy cycles & & & 0.741 \\
\hline$<4$ & 72 & 16 & \\
\hline$\geq 4$ & 260 & 19 & \\
\hline SII & & & $<0.001$ \\
\hline$<660$ & 183 & 30 & \\
\hline$\geq 660$ & 149 & 10 & \\
\hline NLR & & & $<0.001$ \\
\hline$<3.57$ & 195 & 28 & \\
\hline$\geq 3.57$ & 137 & 10 & \\
\hline PLR & & & $<0.001$ \\
\hline$<147$ & 179 & 27 & \\
\hline$\geq 147$ & 153 & 11 & \\
\hline PNI & & & $<0.001$ \\
\hline$\geq 52.95$ & 94 & 36 & \\
\hline$<52.95$ & 238 & 15 & \\
\hline
\end{tabular}

$C R$ complete response, $P R$ partial response, $P D$ progressive disease, $S D$ stable disease, SII systemic immune-inflammation index, NLR neutrophil/lymphocyte ratio, $P L R$ platelet/lymphocyte ratio, $P N /$ prognostic nutritional index

* $p$ log-rank test

in patients with gastric cancer [16]. In another study of 112 patients with hepatocellular carcinoma, patients with 
Table 4 Multivariate analysis of potential factors associated with overall survival in patients with locally advanced NSCLC

\begin{tabular}{llll}
\hline Variables & HR & $\mathbf{9 5 \%} \mathbf{C l}$ & $\boldsymbol{p}$ \\
\hline ECOG PS & & & 0.008 \\
$0-1$ & 1.00 & & \\
2 & 1.744 & $1.158-2.626$ & 0.028 \\
T stage & & & \\
T1-T2 & 1.00 & & 0.018 \\
T3-T4 & 1.332 & $1.032-1.718$ & \\
N stage & & & \\
Negative & 1.00 & & \\
Positive & 1.848 & $1.113-3.068$ & \\
Clinical stage & & & \\
III A & 1.00 & & \\
III B & 1.058 & $0.828-1.350$ & \\
SII & & & \\
$<660$ & 1.00 & & 0.001 \\
$\geq 660$ & 2.105 & $1.481-2.741$ & \\
NLR & & & \\
$<3.57$ & 1.00 & & \\
$\geq 3.57$ & 1.934 & $1.448-2.585$ & \\
PLR & & & \\
$<147$ & 1.00 & $0.933-1.709$ & \\
$\geq 147$ & 1.299 & $0.966-1.748$ & \\
PNI & 1.00 & & \\
$\geq 52.95$ & 1.263 & & \\
$<52.95$ & & & \\
\hline
\end{tabular}

$H R$ hazard ratio, $95 \mathrm{Cl} 95 \%$ confidence interval, SIl systemic immuneinflammation index, NLR neutrophil/lymphocyte ratio, $P L R$ platelet/lymphocyte ratio, $P N /$ prognostic nutritional index

PNI $<45$ were more likely to have portal vein thrombosis and worse Child-Turcotte-Pugh class [17]. In consistent with these earlier results, SII $\geq 660$ were $60 \%$ in T3/4 cases compared with $32 \%$ in $\mathrm{T} 1 / 2$ cases in our results.

Although cisplatin-based concurrent CRT has been the standard treatment option for locally advanced NSCLC, chemotherapy resistance remains the main obstacle in cancer treatment $[18,19]$. Chronic inflammation plays an important role in induction of chemoradiation resistance. In this study, we observed that high SII was associated with chemoradiation resistance in patients with locally advanced NSCLC. Several inflammation-based biomarkers are known to be correlated with treatment response. A recent retrospective study found that high NLR was significantly correlated with chemotherapy resistance in patient with advanced NSCLC treated with first line platinum-based chemotherapy [20]. Similar findings have also been observed by Cho and Mabuchi that tumor-related leukocytosis (TRL) was significantly correlated with poor radiation response in patient with uterine cervical carcinoma [21, 22]. In the retrospective analysis by Mabuchi, TRL $(+)$ patients had upregulated tumor granulocyte colony-stimulating factor (G-CSF) and increased myeloid-derived suppressor cells (MDSCs) in blood. MDSCs are heterogenic and immunosuppressive subpopulation of cells that enhance tumor progression through stimulating vasculogenesis [23]. These results suggested that G-CSF-induced MDSCs stimulated angiogenesis may partly contribute to tumor resistance to radiation.

The prognostic significances of inflammation-based biomarkers have been shown in many solid tumors, most notably in prostate cancer, colorectal cancer, esophageal squamous cell carcinoma, melanoma and NSCLC [24-28]. Like others, we have demonstrated that baseline $\mathrm{SII} \geq 660, \mathrm{NLR} \geq 3.57, \mathrm{PLR} \geq 147$ and $\mathrm{PNI}<52.95$ could predict poor clinical outcomes for the whole cohort of patient with locally advanced NSCLC. SII and NLR remained significantly prognostic even after adjusted for other parameters, such as $\mathrm{T}$ stage, $\mathrm{N}$ stage, ECOG performance status and clinical stage. Moreover, our multivariate analysis showed that SII was superior to NLR in terms of prognostic ability. Our study is not the first to assess SII and cancer patient prognosis. Hu et al. have previously demonstrated the prognostic significance of SII in patients with hepatocellular carcinoma receiving curative resection [14]. The mechanism by which high SII contributes to a poor prognosis in patients with locally advanced NSCLC is unclear. Theories at present focus on the relative neutrophilia, thrombocytosis and lymphopenia that occur as part of systemic inflammation response triggered by cancer. In the present study, SII was calculated as platelet counts $\times$ neutrophil counts/lymphocyte counts. Patients with elevated SII often have thrombocytosis, neutrophilia, and/or lymphopenia. Thrombocytosis, a paraneoplastic syndrome, has been reported in as many as $10-57 \%$ of cancer patients [29]. Recent studies have found a significant role of platelets during tumor development and progression. Indeed, there is increasing evidence that a high level of platelet count is associated with worse survival in patients with cancer [30]. An elevated platelet counts could stimulate tumor angiogenesis and protect tumor cells from cytolysis, thereby contributing to tumor metastasis [31]. Furthermore, neutrophils have also been shown to have tumor-promoting abilities. Relative neutrophilia increases the number of inflammatory factors such as pro-angiogenic factor (VEGF), growth factor (CXCL8), and anti-apoptotic factor (NF$\mathrm{kB})$ which may establish a tumor microenvironment and promote tumor growth and progression [32, 33]. In contrast to neutrophils, lymphocytes have an important role in tumor defence by inducing cytotoxic cell death and inhibiting tumor cell proliferation and migration [34, 
35]. A relative lymphopenia may reflect a lower number of $\mathrm{CD}_{4}^{+} \mathrm{T}$ helper lymphocytes, resulting in a poorer lymphocyte-mediated immune response to malignancies [36]. All of these may promote tumor cells growth, progression and metastasis.

The present study has several limitations despite the demonstration of the prognostic value of SII in patients with stage III NSCLC. First, this is a retrospective analysis; hence there are several potential factors that might have influenced the studied results. Second, data on all patients were collected from a single institute and number of patients is relatively small. Also, the section of treatment modalities and chemotherapy regimens were heterogeneous throughout the period. Therefore, a multiinstitutional investigation, especially a prospective validation study is needed to confirm the results.

\section{Conclusions}

In conclusion, our study has demonstrated that the SII, an inflammation-based prognostic biomarker, is an independent prognostic marker for poor survival in patients with stage III NSCLC. In particular, SII is superior to NLR, PLR and PNI in terms of prognostic ability.

\begin{abstract}
Abbreviations
NSCLC: non-small cell lung cancer; CRT: chemoradiotherapy; NLR: neutrophil to lymphocyte ratio; PLR: platelet to lymphocyte ratio; PNI: prognostic nutritional index; SII: systemic immune-inflammation index; TNM: tumornode-metastasis; ECOG PS: Eastern Cooperative Oncology Group performance status; RECIST: Response Evaluation Criteria in Solid Tumor; CR: complete response; PR: partial response; PD: progressive disease; SD: stable disease; ROC: receiver operating characteristic; OS: overall survival; TRL: tumor-related leukocytosis; MDSCs: myeloid-derived suppressor cells; HR: hazard ratio; Cl: confidence interval.
\end{abstract}

\section{Authors' contributions}

YJS and YST conceived and designed the experiments and were responsible for data analysis and writing the manuscript. JT and XLZ were responsible for providing the clinical samples. YQS was responsible for data collection. All authors read and approved the final manuscript.

\section{Author details \\ ${ }^{1}$ Department of Radiation Oncology, Huai'an First People's Hospital, Nanjing Medical University, Huai'an, Jiangsu, China. ${ }^{2}$ Department of Gerontology, Huai'an First People's Hospital, Nanjing Medical University, Huai'an, Jiangsu, China. ${ }^{3}$ Department of Respiratory Medicine, Huai'an First People's Hospital, Nanjing Medical University, Huai'an, Jiangsu, China.}

\section{Acknowledgements}

The authors acknowledge Qi-Wen Deng for his professional statistical analysis.

\section{Competing interests}

The authors declare that they have no competing interests.

\section{Availability of data and materials}

All data included in the present study were presented in the main manuscript.

\section{Consent for publication}

Informed consent was obtained from all participants for publication.
Ethics approval and consent to participate

The study was approved by the Research Ethics Committee, Nanjing Medical University Huai'an First Hospital. Informed consent was exempted due to the retrospective nature of the study.

\section{Funding}

This project was supported by Grants from The National Nature Science Foundation of China (No. 81602118).

\section{Publisher's Note}

Springer Nature remains neutral with regard to jurisdictional claims in published maps and institutional affiliations.

Received: 14 September 2017 Accepted: 26 October 2017

Published online: 31 October 2017

\section{References}

1. Vansteenkiste J, De Ruysscher D, Eberhardt WE, Lim E, Senan S, Felip E, et al. Early and locally advanced non-small-cell lung cancer (NSCLC): ESMO Clinical Practice Guidelines for diagnosis, treatment and follow-up. Ann Oncol. 2013;24(Suppl 6):vi89-98.

2. NSCLC Meta-analysis Collaborative Group. Preoperative chemotherapy for non-small-cell lung cancer: a systematic review and meta-analysis of individual participant data. Lancet. 2014;383(9928):1561-71.

3. Stinchcombe TE, Zhang Y, Vokes EE, Schiller JH, Bradley JD, Kelly K, et al. Pooled analysis of individual patient data on concurrent chemoradiotherapy for stage III non-small-cell Lung cancer in elderly patients compared with younger patients Who participated in US National Cancer Institute Cooperative Group Studies. J Clin Oncol. 2017;35(25):2885-92.

4. Antoni D, Mornex F. Chemoradiotherapy of locally advanced nonsmall cell lung cancer: state of the art and perspectives. Curr Opin Oncol. 2016;28(2):104-9.

5. Pless M, Stupp R, Ris H-B, Stahel RA, Weder W, Thierstein S, et al. Induction chemoradiation in stage IIIA/N2 non-small-cell lung cancer: a phase 3 randomised trial. Lancet. 2015;386(9998):1049-56.

6. Feng W, Fu XL, Cai XW, Yang HJ, Wu KL, Fan M, et al. Patterns of localregional failure in completely resected stage IIIA(N2) non-small cell lung cancer cases: implications for postoperative radiation therapy clinical target volume design. Int J Radial Oncol Biol Phys. 2014;88(5):1100-7.

7. Fortunato $\mathrm{O}$, Boeri M, Moro M, Verri C, Mensah M, Conte D, et al. Mir-660 is downregulated in lung cancer patients and its replacement inhibits lung tumorigenesis by targeting MDM2-p53 interaction. Cell Death Dis. 2014;5:e1564

8. Proctor MJ, Morrison DS, Talwar D, Balmer SM, O'Reilly DS, Foulis AK, et al. An inflammation-based prognostic score (mGPS) predicts cancer survival independent of tumour site: a Glasgow Inflammation Outcome Study. Br J Cancer. 2011;104(4):726-34.

9. Proctor MJ, Morrison DS, Talwar D, Balmer SM, Fletcher CD, O'Reilly DS, et al. A comparison of inflammation-based prognostic scores in patients with cancer. A Glasgow Inflammation Outcome Study. Eur J Cancer. 2011:47(17):2633-41.

10. Cannon NA, Meyer J, lyengar P, Ahn C, Westover KD, Choy H, et al. Neutrophil-lymphocyte and platelet-lymphocyte ratios as prognostic factors after stereotactic radiation therapy for early-stage non-small-cell lung cancer. J Thoaoc Oncol. 2015;10(2):280-5.

11. Shimizu K, Okita R, Saisho S, Yukawa T, Maeda A, Nojima Y, et al. Prognostic nutritional index before adjuvant chemotherapy predicts chemotherapy compliance and survival among patients with non-small-cell lung cancer. Ther Clin Risk Manag. 2015;11:1555-61.

12. Kinoshita A, Onoda H, Imai N, Iwaku A, Oishi M, Fushiya N, et al. Comparison of the prognostic value of inflammation-based prognostic scores in patients with hepatocellular carcinoma. Br J Cancer. 2012;107(6):988-93.

13. Minna JD, Sheng J, Yang Y-P, Ma Y-X, Qin T, Hu Z-H, et al. Low prognostic nutritional index correlates with worse survival in patients with advanced NSCLC following EGFR-TKIs. PLOS ONE. 2016;11(1):e0147226. 
14. Hu B, Yang XR, Xu Y, Sun YF, Sun C, Guo W, et al. Systemic immune-inflammation index predicts prognosis of patients after curative resection for hepatocellular carcinoma. Clin Cancer Res. 2014;20(23):6212-22.

15. Peng Y, Li Z, Zhang S, Xiong Y, Cun Y, Qian C, et al. Association of DNA base excision repair genes (OGG1, APE1 and XRCC1) polymorphisms with outcome to platinum-based chemotherapy in advanced nonsmall-cell lung cancer patients. Int J Cancer. 2014;135(11):2687-96.

16. Deng Q, He B, Liu X, Yue J, Ying H, Pan Y, et al. Prognostic value of preoperative inflammatory response biomarkers in gastric cancer patients and the construction of a predictive model. J Transl Med. 2015;13:66.

17. Pinato DJ, North BV, Sharma R. A novel, externally validated inflammationbased prognostic algorithm in hepatocellular carcinoma: the prognostic nutritional index (PNI). Br J Cancer. 2012;106(8):1439-45.

18. Liu J, Wan L, Lu K, Sun M, Pan X, Zhang P, et al. The long noncoding RNA MEG3 contributes to cisplatin resistance of human lung adenocarcinoma. PLoS ONE. 2015;10(5):e0114586.

19. Warner A, Dahele M, Hu B, Palma DA, Senan S, Oberije C, et al. Factors associated with early mortality in patients treated with concurrent chemoradiation therapy for locally advanced non-small cell lung cancer. Int J Radial Oncol Biol Phys. 2016;94(3):612-20.

20. Yao Y, Yuan D, Liu H, Gu X, Song Y. Pretreatment neutrophil to lymphocyte ratio is associated with response to therapy and prognosis of advanced non-small cell lung cancer patients treated with first-line platinum-based chemotherapy. Cancer Immunol Immunother. 2013;62(3):471-9.

21. Cho Y, Kim KH, Yoon HI, Kim GE, Kim YB. Tumor-related leukocytosis is associated with poor radiation response and clinical outcome in uterine cervical cancer patients. Ann Oncol. 2016;27(11):2067-74.

22. Mabuchi S, Matsumoto Y, Kawano M, Minami K, Seo Y, Sasano T, et al. Uterine cervical cancer displaying tumor-related leukocytosis: a distinct clinical entity with radioresistant feature. J Natl Cancer Inst. 2014;106(7):2067-2074.

23. Deng Z, Rong Y, Teng Y, Zhuang X, Samykutty A, Mu J, et al. Exosomes miR-126a released from MDSC induced by DOX treatment promotes lung metastasis. Oncogene. 2017;36(5):639-51.

24. van Soest RJ, Templeton AJ, Vera-Badillo FE, Mercier F, Sonpavde G, Amir E, et al. Neutrophil-to-lymphocyte ratio as a prognostic biomarker for men with metastatic castration-resistant prostate cancer receiving firstline chemotherapy: data from two randomized phase III trials. Ann Oncol. 2015;26(4):743-9.

25. Guthrie GJ, Roxburgh CS, Farhan-Alanie OM, Horgan PG, McMillan DC Comparison of the prognostic value of longitudinal measurements of systemic inflammation in patients undergoing curative resection of colorectal cancer. Br J Cancer. 2013;109(1):24-8.

26. Gebhardt C, Sevko A, Jiang H, Lichtenberger R, Reith M, Tarnanidis K, Holland-Letz T, Umansky L, Beckhove P, Sucker A, et al. Myeloid cells and related chronic inflammatory factors as novel predictive markers in melanoma treatment with ipilimumab. Clin Cancer Res. 2015;21(24):5453-9.

27. Pinato DJ, Shiner RJ, Seckl MJ, Stebbing J, Sharma R, Mauri FA. Prognostic performance of inflammation-based prognostic indices in primary operable non-small cell lung cancer. Br J Cancer. 2014;110(8):1930-5.

28. Sharaiha RZ, Halazun KJ, Mirza F, Port JL, Lee PC, Neugut Al, et al. Elevated preoperative neutrophil:lymphocyte ratio as a predictor of postoperative disease recurrence in esophageal cancer. Ann Surg Oncol. 2011;18(12):3362-9.

29. Wojtukiewicz MZ, Sierko E, Hempel D, Tucker SC, Honn KV. Platelets and cancer angiogenesis nexus. Cancer Metastasis Rev. 2017;36(2):249-62.

30. Gao L, Zhang H, Zhang B, Zhang L, Wang C. Prognostic value of combination of preoperative platelet count and mean platelet volume in patients with resectable non-small cell lung cance. Oncotaret. 2017;8(9):15362-641.

31. Li N, Yu Z, Zhang X, Liu T, Sun YX, et al. Elevated mean platelet volume predicts poor prognosis in colorectal cancer. Sci Rep. 2017;7(1):10261.

32. Scilla KA, Bentzen SM, Lam VK, Mohindra P, Nichols EM, Vyfhuis MA, et al. Neutrophil-lymphocyte ratio is a prognostic marker in patients with locally advanced (stage IIIA and IIIB) non-small cell lung cancer treated with combined modality therapy. Oncologist. 2017;22(6):737-42.

33. Paramanathan A, Saxena A, Morris DL. A systematic review and metaanalysis on the impact of pre-operative neutrophil lymphocyte ratio on long term outcomes after curative intent resection of solid tumours. Surg Oncol. 2014;23(1):31-9.

34. Zhou XL, Li YQ, Zhu WG, Yu CH, Song YQ, Wang WW, et al. Neutrophilto-lymphocyte ratio as a prognostic biomarker for patients with locally advanced esophageal squamous cell carcinoma treated with definitive chemoradiotherapy. Sci Rep. 2017;7:42581.

35. Kang MH, Go Sl, Song HN, Lee A, Kim SH, Kang JH, et al. The prognostic impact of the neutrophil-to-lymphocyte ratio in patients with small-cell lung cancer. Br J Cancer. 2014;111(3):452-60.

36. Keizman D, Gottfried M, Ish-Shalom M, Maimon N, Peer A, Neumann $A$, et al. Pretreatment neutrophil-to-lymphocyte ratio in metastatic castration-resistant prostate cancer patients treated with ketoconazole: association with outcome and predictive nomogram. Oncologist. 2012;17(12):1508-14.

\section{Submit your next manuscript to BioMed Central and we will help you at every step:}

- We accept pre-submission inquiries

- Our selector tool helps you to find the most relevant journal

- We provide round the clock customer support

- Convenient online submission

- Thorough peer review

- Inclusion in PubMed and all major indexing services

- Maximum visibility for your research

Submit your manuscript at www.biomedcentral.com/submit
() Biomed Central 